\title{
«Envejecer es cambiar»: La institucionalización de la geriatría y la evolución del concepto de vejez durante el franquismo
}

\author{
Emilia Martos Contreras (*) \\ $\left.{ }^{*}\right)$ orcid.org/0000-0003-1705-5721. Departamento de Geografía, Historia y Humanidades. \\ Universidad de Almería.emiliamartoscontreras@gmail.com
}

Dynamis

[0211-9536] 2019; 39 (2): 453-475

http://dx.doi.org/10.30827/dynamis.v39i2.9843
Fecha de recepción: 18 de agosto de 2018

Fecha de aceptación:10 de febrero de 2019

SUMARIO: 1.-Introducción. 2.-Posguerra y vejez. 2.1.-El nacimiento de la disciplina geriátrica en España. 2.2.-El cambio en la comprensión de la vejez. 3.-La institucionalización de la geriatría y su desarrollo político en el segundo franquismo. 4.- Conclusiones.

RESUMEN: El concepto de vejez evolucionó significativamente a lo largo del siglo XX. A ello contribuyó, entre otras cuestiones, la aparición de la geriatría. Los defensores de esta nueva disciplina médica señalaron que la vejez no debía entenderse como sinónimo de enfermedad, sino que era un periodo más de la vida. Además, plantearon el tratamiento pluridisciplinar como un requisito básico para una correcta atención a las necesidades específicas de las personas mayores. Algunos médicos de la España franquista afrontaron con entusiasmo estos nuevos conocimientos e intentaron expandirlos desde mediados de siglo. Sin embargo, el sistema franquista condicionó de manera particular este proceso. Aunque aprobó normas encaminadas a proteger a las personas mayores, en la práctica no resolvió ninguno de los numerosos problemas que sufrió este colectivo. Esto conllevó un creciente distanciamiento entre la dura realidad y la evolución de la comprensión de la vejez y los derechos de las personas mayores.

PALABRAS CLAVE: Franquismo, vejez, geriatría, personas mayores, historia social.

KEYWORDS: Francoism, old age, geriatrics, elderly people, social history. 


\section{Introducción $(*)$}

La evolución de la medicina durante el franquismo fue determinada, principalmente, por el intento de acabar con la tradición republicana y la influencia del nacional-catolicismo. Particularmente los años de posguerra fueron un punto de inflexión, tanto por el penoso contexto heredado de la contienda como la dura realidad que supuso la implantación del nuevo Estado dictatorial y sus premisas ideológicas ${ }^{1}$. Como en el resto de ciencias, en medicina el fin de la guerra supuso retroceder a las concepciones de principios de siglo, además de la obligación de asimilar las consignas franquistas y las normas arbitrarias propias de los sistemas dictatoriales ${ }^{2}$. Sin embargo, a pesar de este claro deterioro, diferentes trabajos de investigación han mostrado que no podemos simplificar los largos años del franquismo a un mero estancamiento o retroceso $^{3}$. Desde el inicio de la contienda, los profesionales sanitarios se concentraron en afrontar las numerosas problemáticas derivadas de la misma, y desde pronto encontramos facultativos que comenzaron a organizarse para retomar su trabajo investigador ${ }^{4}$. Por otra parte, a pesar de la autarquía

(*) Este trabajo ha sido realizado en el ámbito del Grupo de Investigación «Estudios del Tiempo Presente» (PAI HUM-756) y del Centro de Investigación «Comunicación y Sociedad» de la Universidad de Almería (Cysoc), y forma parte del proyecto I+D «Políticas sociales e instituciones locales en el tardo franquismo y la Transición. La lucha por la democracia en municipios de Andalucia (1963-1986)», financiado por el Ministerio de Economía y Competitividad (Ref. HAR2017-83744-C3-3-P).

1. La nueva realidad socio-política tuvo una influencia negativa sobre las ciencias en general. Véase, por ejemplo, Otero Carvajal, Luis Enrique. La destrucción de la ciencia en España. Las consecuencias del triunfo militar de la España franquista. Historia y Comunicación Social. 2001; 6: $149-186$.

2. Según Marset, Saéz y Martínez la victoria del franquismo implicó un abandono de las dimensiones sociales y de las nuevas concepciones sobre epidemiología y administración sanitaria, en favor de la práctica de integrar la higiene y la microbiología. Marset Campos, Pedro; Sáez Gómez, José Miguel; Martínez Navarro, Fernando. La Salud Pública durante el franquismo. Dynamis, 1995; 15: 219. En cuanto a la influencia de la dictadura sobre el desarrollo sanitaria véase, entre otros, Carasa Soto, Pedro. La Revolución Nacional-Asistencial durante el primer franquismo (1936-1940). Historia Contemporánea, 1997; 16: 37-47 y Ortiz Heras, Manuel; González Madrid, Damián A. Las políticas sociales en la España del siglo XX. De la dictadura franquista a la transición. In: Porras Gallo, María Isabel, ed. La erradicación y el control de las enfermedades infecciosas. Madrid: La Catarata; 2016.

3. Véase como ejemplo reciente: Campos, Ricardo; González de Pablo, Ángel. Psiquiatría en el primer franquismo: saberes y prácticas para un «Nuevo Estado». Dynamis. 2017; 37 (1): 13-21.

4. Un buen ejemplo de este planteamiento sería la trayectoria del doctor Gregorio Marañón, quién en 1945 volvió del exilió, retomando a continuación su carrera profesional y académica. López Vega, Antonio. La Universidad de Marañón. Cuadernos del Instituto Antonio Nebrija. 2004; 7: 73. 
del periodo y las diversas limitaciones, no podemos negar la influencia de planteamientos internacionales en el desarrollo de la medicina española ${ }^{5}$. En los primeros años, sobre todo en torno a la Falange, se puso la vista sobre los avances sociosanitarios implementados en Italia y Alemania ${ }^{6}$. Sin embargo, no fue hasta 1951, con la entrada de España en la Organización Mundial de la Salud cuando realmente empezó a apreciarse la llegada de nuevos debates ${ }^{7}$. Otra de las vías de contacto con el exterior fueron las organizaciones internacionales como Cáritas y Cruz Roja, que divulgaron sus planteamientos a través de la acción, seminarios y publicaciones como Documentación Social. Por lo tanto, España no fue impermeable a las tendencias internacionales y el Estado, más aún en su intento de modernización y supervivencia, aceptó parte de los planteamientos que le indicaban los profesionales y los colectivos interesados ${ }^{8}$. No obstante, una cosa fue aceptar las premisas y otra muy diferente hacer un plan efectivo para ponerlas en marcha. De hecho, las

Sobre la continuidad del Cuerpo Nacional de Sanidad véase Molero Mesa, Jorge; Jiménez Lucena, Isabel. Salud y burocracia en España. Los cuerpos de Sanidad Nacional (1855-1951). Revista Española de Salud Pública, 2000; 74: 46-53. Como ejemplo de reorganización profesional puede verse el caso de la psiquiatría descrita en el siguiente trabajo Huertas, Rafael. En los inicios de la psiquiatría franquista. El Congreso Nacional de Neurología y Psiquiatría (Barcelona, 1942). Dynamis. 2017; 37(1): 23-43.

5. Existen diferentes trabajos que se han centrado en ese aspecto, véase, por ejemplo, Barona Vilar, Josep Lluis; Bernabeu-Mestre, Josep. La salud y el Estado, el movimiento sanitario internacional y la administración española (1815-1945). Valencia: Universidad de Valencia; 2008.

6. Molero Mesa, Jorge. Enfermedad y previsión social en España durante el primer franquismo (1936-1951). Dynamis. 1994; 14: 199-225.

7. Diferentes trabajos han mostrado como parte de las ideas planteadas por la organización mundial fueron debatidas en España, véase, por ejemplo: Terrón Bañuelos, Aida; Comelles Josep Maria; Perdiguero Gil, Enrique. Schools and health education in Spain during the dictatorship of General Franco (1939-1975). History of Education Review. 2017; 46 (2): 208-223; Perdiguero Gil, Enrique; Comelles, Josep Maria; Terrón Bañuelos, Aida. La introducción de las ciencias sociomédicas en el ámbito de la educación sanitaria y la educación médica durante el franquismo. In: Zarzoso Orellana, Alfons; Arrizabalaga Valbuena, Jon, eds. Al servicio de la Salud Humana. La historia de la Medicina ante los retos del siglo XXI. Sant Feliu de Guíxols: Sociedad Española de Historia de la Medicina, 2017, p. 123-128.

8. La diversidad funcional es uno de los ámbitos en los que mejor se puede ver esa correspondencia con el desarrollo internacional. Véase, especialmente, Martínez Pérez, José; del Cura González, Mercedes. El «llamamiento del deber»: influencia exterior, interés del Estado y modernización de las estrategias de gestión de la discapacidad en España (1956-1970). In: González Madrid, Damián; Ortiz Heras, Manuel; Pérez Garzón, Juan Sisinio, eds. La historia, lost in translation? Actas del XIII Congreso de la Asociación de Historia Contemporánea. Cuenca: Universidad de Castilla La Mancha; 2017, p. 1775-1786. 
políticas sociales del franquismo fueron hasta su último momento ineficaces e insuficientes, lo que llevó a una creciente protesta social.

Desde la historia social no solo se han investigado las diferentes vías de oposición al franquismo. También se han analizado las dinámicas de desgaste que pudiesen justificar la creciente acción social del último periodo dictatorial y el devenir de los sucesos que acompañaron a la muerte de Franco. Coincidiendo con los últimos trabajos que se han hecho sobre ese periodo histórico compartimos la consideración de que las bases sociales jugaron un papel importante en la evolución política y social de España. De tal forma que el destino del país no estuvo exclusivamente guiado y programado por una élite política selecta. Partiendo de este planteamiento teórico, en los últimos años, han aumentado las aportaciones que, desde diferentes perspectivas, examinan algunos de los ángulos del complejo proceso de cambio político ${ }^{9}$. En esa dirección se encamina nuestra línea de investigación, que se centra en los profesionales del ámbito de lo social y su papel como posibles agentes de transformación. Según nuestra hipótesis de trabajo, en contacto con la realidad más deprimida del franquismo e influidos teóricamente por el desarrollo académico internacional, los profesionales de diferentes disciplinas defendieron una serie de cambios que, de forma directa o indirecta, allanaron el camino hacia la democratización o, al menos, ayudaron a desgastar el sistema ${ }^{10}$. En ese sentido, nos ha parecido de especial interés acercarnos a la temática de la geriatría y sus intentos de implantación en España. Como veremos, su defensa surgió por parte de profesionales completamente integrados en el sistema dictatorial. Sin embargo, eso no impidió que, a través de sus declaraciones, las premisas de la nueva disciplina geriátrica acabaran derivando hacía una reflexión crítica respecto a la situación socio-económica de las personas mayores en España y, por ende, sumándose al creciente espíritu crítico de la década de los sesenta y setenta. El objetivo de nuestro

9. Esta interpretación ya fue planteada por Maravall, José María. La política de la transición, 19751980. Madrid: Taurus; 1982. Sin embargo, no ha adquirido una mayor relevancia hasta la primera década del siglo XXI. Véase, por ejemplo, Ortiz Heras, Manuel ed. Movimientos sociales en la crisis de la dictadura y la Transición: Castilla la Mancha, 1969-1979. Ciudad Real: Almud; 2008 y Quirosa-Cheyrouze y Muñoz, Rafael ed. La sociedad española en la Transición: los movimientos sociales en el proceso democratizador. Madrid: Biblioteca Nueva; 2011.

10. Véase Martos Contreras, Emilia. El trabajo social y la construcción de la democracia: una mirada desde la periferia. In: Fernández Amador, Mónica; Quirosa-Cheyrouze y Muñoz, Rafael; coord. Movimientos sociales e instituciones locales en la transición: la lucha por la democracia en la Andalucía rural. Madrid: Catarata; 2018, p. 251-279. 
trabajo es rastrear estos planteamientos y señalar el progresivo desequilibrio entre la realidad social de las personas mayores y las propuestas de mejora que el propio Estado defendía. Además, proponemos una pequeña incursión en los medios de comunicación escritos para sopesar la difusión de la temática. Consideramos que esta evolución es de especial interés para entender sucesos posteriores, como por ejemplo las importantes movilizaciones de pensionistas y jubilados de los años setenta. Con todo, queremos advertir que no pretendemos, pues no es objeto de este trabajo, crear una línea de causalidad directa con estos acontecimientos posteriores. De hecho, debido a las limitaciones propias de este tipo de aportaciones, nuestra atención se centrará exclusivamente en los profesionales médicos que se interesaron por esta disciplina y las implicaciones que tuvo la aparición de una nueva comprensión de la vejez en un contexto adverso. Para ello hemos utilizado como fuentes primarias declaraciones de los profesionales, informes de la época, revistas especializada y prensa general.

\section{Posguerra y vejez}

La guerra civil y los duros años posteriores fueron especialmente difíciles para las personas mayores, un colectivo vulnerable a la pobreza y las carencias del sistema sanitario y asistencial. Ya desde la proclamación del Fuero de los Españoles, la dictadura hizo una referencia expresa a la «asistencia a la vejez» ${ }^{11}$. Sin embargo, como trataremos, el franquismo no llegó nunca a resolver las necesidades asistenciales de este colectivo, ni siquiera, después de la aprobación de la Ley de Bases de la Seguridad Social de 1963. Las principales políticas sociales de la dictadura fueron de tipo retributivo. En el primero periodo franquista hay que destacar la creación, en 1939, del Subsidio de Vejez, y su evolución, en 1947, hacia el Seguro Obligatorio de la $V_{e j e z}{ }^{12}$. Esta aprobación supuso una importante extensión de los asegurados, aunque su incidencia real fue muy escasa debido, principalmente, a la baja cuantía de las pensiones ${ }^{13}$. Igualmente, hubo otras prestaciones económicos

11. Artículo 28, Ley de 17 de julio de 1945 que aprueba el Fuero de los Españoles. BOE. 18 Jul 1945.

12. Véase Ignasi Camós, Victoria. La configuración de la prestación de jubilación en el régimen contributivo de la seguridad social. Gerona: Universitat de Girona; 2001.

13. El número de asegurados aumentó en la década de los cuarenta en casi un millón, y la inversión en pesetas pasó de 9 millones a más de 77 millones. Sin embargo, incluso después de la 
puntuales, como el reconocimiento de los ascendientes que habitasen en el núcleo familiar de un trabajador ${ }^{14}$.

En el aspecto asistencial, como ya dijimos, hubo muy pocas aportaciones. El Auxilio Social, como principal agente asistencial del primer franquismo, en los años de la posguerra estuvo ocupado en otras necesidades. En consecuencia su atención a las personas mayores fue más bien coyuntural y básica, dándose principalmente a través de los comedores sociales ${ }^{15}$. Por su parte la Dirección General de Beneficencia inició una lenta construcción de algunas residencias, aunque fueron las Diputaciones Provinciales - obligadas por la Ley de Régimen Local- las que mantuvieron los centenarios Asilos Provinciales, donde se acogían a personas mayores en estado muy necesitado y de abandono absoluto. Pero, en la práctica, el agente asistencial principal fue la Iglesia, ya fuese en colaboración con las instituciones públicas o a través de los múltiples asilos particulares. Algunas congregaciones religiosas estaban especialmente centradas en el cuidado de mayores, como fue el caso de la Congregación de las Hermanas de los Ancianos Desamparados ${ }^{16}$. Las religiosas se entregaban a su labor, tanto en las instituciones privadas como en las públicas, sin sueldo ni horario laboral y teniendo que lidiar con la constante falta de recursos. En 1963, había en España 418 asilos, de las cuales solo 86 pertenecían a la beneficencia pública. Según los datos de la época, se estimaba una carencia de unas 43.000 camas, a la que se sumarían las profundas deficiencias en las plazas que ya existentes ${ }^{17}$.

Los asilos eran herederos directos, en fondo y forma, de las primeras instituciones de atención a la vejez aparecidas en siglos anteriores. Los centros caritativos de la dictadura tuvieron, por su intermitente sistema de financiación y el alcance de sus obligaciones, graves problemas económicos que explican parte de las múltiples carencias que sufrieron. Según una publi-

subida de 1948, la pensión no superaba las 200 pesetas mensuales. Piédrola Gil, Gonzalo. La Gerocultura, especialidad nueva en Sanidad Nacional. Su estudio, necesidad y organización. Madrid: Dirección General de Sanidad; 1955, p. 56.

14. A través del llamado «Plus Familiar». Orden de 29 de marzo de 1946. Por supuesto, dentro de la tradicional feminización de la asistencia potenciada por el franquismo, el cuidado recaía sobre las mujeres.

15. Cenarro Lagunas, Ángela. La sonrisa de Falange. Auxilio Social durante la Guerra Civil y la posguerra. Barcelona: Crítica; 2006.

16. Véase Salgado Alba, Alberto. Ayuda Social a Ancianos. In: VAA. Problemas fundamentales de beneficencia y asistencia social. Madrid: Ministerio de la Gobernación; 1966, p. 189.

17. Couceiro Núñez, Enrique. Encuesta sobre los Asilos en España. Documentación Social. 1963; 20: 91. 
cación del Ministerio de Gobernación de 1966: «Las condiciones higiénicas y sanitarias son deficientes en la mayoría de los asilos de nuestra patria» ${ }^{18}$. Estos centros estuvieron generalmente situados en edificios centenarios en los que los dormitorios de las personas mayores eran grandes salas colectivas divididas por sexo. En raras ocasiones los asilos contaban con dormitorios más reducidos u opciones para albergar conjuntamente a los matrimonios. Así que al trauma de mudarse al asilo se sumaba el pesar de la separación física de los cónyuges. Además, la vida en estos centros era muy austera y se regía por unas normas estrictas que apenas tenían en cuanta los deseos y afanes de los $\operatorname{acogidos}^{19}$. Así lo recogía un diario de 1971:

«El asilo, desgraciadamente, dados nuestros pobres conceptos del amor al prójimo y de la justicia, se había convertido en algo cuyo "disfrute" repugnaba a aquellos mismos a quienes debía favorecer. Un régimen entre penitenciario, conventual y cuartelero, con sus limitaciones de libertad, sus exigencias confesionales, sus dormitorios colectivos, la separación de los matrimonios, los horarios absurdos, la carencia de distracciones, de bibliotecas, las limitaciones de comunicación social, y tantos etcéteras más, algo poco menos que indeseado. Y, sin embargo, era a estos establecimientos a los que se veían abocados cuantos, carentes de recursos y cargados de años, no tenían otra opción» ${ }^{20}$.

Según la concepción tradicional, la vejez era poco más que la antesala de la muerte, y esto, visto desde una óptica religiosa, conllevaba que se le diese a la espiritualidad una importancia mucho mayor que la que se le otorgaba a la mundanidad y al bienestar del cuerpo. Este abandono de lo físico ayudaba a reforzar el estereotipo del «anciano pobre y desvalido». En definitiva, un ser pasivo en todos los sentidos, infantilizado y más necesitado que nadie de la caridad social y la ayuda familiar ${ }^{21}$. En este contexto, la llegada de la geriatría, y su nueva concepción de la vejez, fue un punto de inflexión que

\footnotetext{
18. Salgado, n. 16, p. 190.

19. Martos Contreras, Emilia. Personas mayores y diversidad funcional física e intelectual durante la transición a la democracia. Almería: Universidad de Almería; 2016, p. 781.

20. Firmado con el pseudónimo «Un donostiarra». Residencias de jubilados. Hoja del Lunes de San Sebastián. 18 Oct 1971, citado en VVAA, Informe-Resumen Nacional sobre la situación de las personas de edad en España. Revista de Seguridad Social. 1982; 16: 516.

21. Aunque podríamos citar muchos ejemplos filmográficos, creemos que es especialmente paradigmática para el estereotipo que venimos describiendo la película El cochecito, de 1960, dirigida por Marco Ferreri y protagonizada por José Isbert.
} 
puso en entredicho tanto la atención médica como la política social a las personas mayores.

\subsection{El nacimiento de la disciplina geriátrica en España}

El término «geriatría» fue acuñado por Ignatz Lewn Nasher en 1909, año en el que creó un Departamento de Geriatría en Estados Unidos. Dos décadas después, Marjory Warren asentó los principios de esta disciplina, y en 1940 la incorporó al Middlessex Hospital de Londres. En 1946 el National Health Service inglés creó la especialidad de geriatría ${ }^{22}$. En principio, la disciplina nació como una rama centrada en tratar las enfermedades de las personas mayores, aunque muy pronto se desarrolló hacía una concepción integral de la asistencia. Su gran novedad radicaba en considerar que la vejez en sí, no era una enfermedad, sino un ciclo vital más. Los primeros geriatras descubrieron que, con los cuidados adecuados, las personas mayores podían mejorar su estado de salud general. Según los principios que se formularon, esta mejoría ocurría cuando se comenzaba a atender a las personas por su enfermedad y se dejaba de ignorarlas por su edad ${ }^{23}$. Por lo tanto, se trataba de un modelo de comprensión renovado, que conllevó la creación de instituciones específicas basadas en conceptos pluridisciplinares. Así, a parte de los servicios geriátricos dentro de los hospitales y las consultas externas especializadas, surgirían nuevos centros como los hospitales de día, y servicios como la ayuda a domicilio. Además, se empezó a hablar de un envejecimiento activo y de la importancia de esta actitud para el bienestar general.

España se incorporó con rapidez a esa nueva tendencia médica. Según Fernando Jiménez Herrero, desde los años cuarenta, la geriatría ya se planteaba como una «rama nueva de la Medicina del futuro», en la Real Academia Nacional de Medicina, donde tuvieron repercusión tanto las ideas de I. L. Nasher como la de otros escritores franceses, ingleses y norteamericanos ${ }^{24}$. De hecho, ya desde mediados de los años cuarenta se empezaron a dar los

\footnotetext{
22. Macías Núñez, Juan Francisco, ed. Geriatría desde el principio. Barcelona: Glosa; 2005, p. 104.

23. Véase Rodríguez Domínguez, Sandalio, La vejez: historia y actualidad. Salamanca: Universidad de Salamanca; 1989 y Salgado Alba, Alberto. Geriatría. Historia, definición, objetivos y fines. Errores conceptuales. Madrid: Medicine; 1983.

24. Jiménez Herrero, Fernando. Evolución e impulsos a la geriatría española aportados por Alberto Salgado Alba. Revista Española de Geriatría y Gerontología. 2001; 36: 20.
} 
primeros y significativos pasos para la instauración y desarrollo de la disciplina en el país. Uno de los pioneros en este ámbito fue Mariano Pañella Casas - médico de la Beneficencia Municipal de Barcelona- que en 1945 fundó el primer servicio geriátrico hospitalario de España en el Hospital Nuestra Señora. En la misma línea, el prestigioso médico y catedrático Manuel Beltrán Báguena impulsó en 1946 el primer dispensario geriátrico. Un servicio de consulta externa que tenía como objetivo la asistencia médica y social ambulatoria a las personas mayores. Inicialmente, dicho dispensario se sitúo en un espacio cedido por la Facultad de Medicina de Valencia, donde el médico y profesor trabajaba. En 1947, y apenas un año después de la instauración de la especialidad de Geriatría en Reino Unido, el mismo Beltrán Báguena encabezó la creación de la primera Cátedra de Geriatría en Valencia, siendo pionero en la enseñanza oficial y universitaria de esta disciplina ${ }^{25}$.

El entusiasmo por la geriatría se concretó el 17 de mayo de 1948 con la fundación en Madrid de la Sociedad Española de Gerontología, cuyo presidente fue de nuevo Beltrán Báguena ${ }^{26}$. Ese año, el mismo Gregorio Marañón mostró también su interés por la disciplina y creó el Departamento de Geriatría del Instituto de Patología Médica del Hospital General de Madrid ${ }^{27}$. Para su inauguración se celebró la Semana Geriátrica, un evento formativo en el que participaron los principales impulsores de la nueva materia ${ }^{28}$. Dos años después, en 1950, se organizó en Barcelona el I Congreso Nacional de Geriatría $^{29}$. Ese mismo año, Bélgica fue el escenario del llamado I Congreso Internacional de Lieja, donde se fundó la Sociedad Internacional de Gerontología. Entre los pocos países representados se encontraba España, que envió como delegado a Pañella Casas ${ }^{30}$. En dicho evento se concretó una de las claves fundamentales de la disciplina: la convicción de que el estudio bioló-

25. De estas fechas datan sus dos obras sobre geriatría: Beltrán Báguena, Manuel, Lecciones de Geriatría: enfermedades de la vejez. Valencia: Editorial Saber; 1947-1948 y Prevención de la vejez achacosa y cuidado de los ancianos. Barcelona: Editorial Científico-Médica; 1949.

26. Arquiola Llopis, Elvira. El envejecimiento en la Medicina Española Contemporánea. Asclepio. 1995; 47: 14.

27. Según Jiménez Herrero se creó «con más buena voluntad que medios». Jiménez, n. 24, p. 20.

28. Curso médico sobre la vejez. ABC. 20 Nov 1948: 13. Sobre el evento véase Vega Díaz, F. Semana Geriátrica en el Instituto de Patología Médica. In: VVAA. Siete Conferencias sobre Geriatría. Madrid: Escelicer; 1950, p. 2-7.

29. Congreso Nacional de Geriatría. La Vanguardia. 16 Jun 1950: 16.

30. Regreso de los delegados en el Congreso de Gerontología. ABC, 11 Ago 1950: 8. 
gico y médico de la vejez no era suficiente, sino que debía completarse con los aspectos psicológicos y sociales, máxima que consolidó la gerontología ${ }^{31}$.

En 1953 nació el Dispensario Geriátrico de la Cruz Roja en su Hospital Central de Madrid. Esta institución se convertiría en un verdadera referente, evolucionando en las siguientes décadas hasta ser el primer hospital geriátrico del país ${ }^{32}$. Al frente de este proyecto estuvo Alberto Salgado, un joven médico que se erigiría como el verdadero representante de la implantación geriátrica en España ${ }^{33}$.

Como vemos, España dio los primeros pasos en la geriatría a la par que otros países occidentales. Sin embargo, no debemos perder la visión de conjunto. A pesar de la temprana adhesión de algunos profesionales a la geriatría, en la práctica, la disciplina tardó mucho en desarrollarse. Como señala el catedrático Ribera Casado, la historia de la geriatría en España ha sido «rica en actividad, intermitente en sus logros, escasa en su implantación asistencial y tremendamente laboriosa en el camino hacia su reconocimiento oficial como especialidad autónoma por parte de la Administración» ${ }^{34}$. De hecho, no fue hasta mucho después, ya en 1978, cuando la especialidad se aprobó definitivamente. No obstante, a pesar de esta tardanza, creemos que su temprana aparición en España sí tuvo una cierta influencia sobre el desarrollo social. En primer lugar, y tal como observaremos, consideramos que se puede rastrear su repercusión en las medidas sanitarias implementadas a partir de los años sesenta. Por otra parte, también sostenemos que influyó en la evolución social del concepto de la vejez, así como en la concepción de los derechos de las personas mayores.

31. Geriatría es la especialidad médica que trata las enfermedades de la población mayor de 65 años, mientras que la gerontología es la ciencia que estudia el proceso y los problemas del envejecimiento desde el ámbito biológico, psicológico y social. Sobre el evento de Lieja véase Shock, N. W. y Baker, G. T. The international Association of Gerontology: A Chronicle,1950-1986. Nueva York: Sprigner Publishing; 1988.

32. Díaz de la Peña, Juan. Estudio del primer dispensario geriátrico médico-social español. Madrid: Universidad Complutense de Madrid; 1990.

33. Sobre sus inicios en la disciplina véase Jiménez, n. 24, p. 21.

34. Ribera Casado, José Manuel. Comisión Nacional de la Especialidad de Geriatría (CNEG): breve reseña histórica. In: Gil Gregorio, Pedro, coord. Manual del residente en Geriatría. Madrid: Sociedad Española de Geriatría y Gerontología; 2011, p. 6. 


\subsection{El cambio en la comprensión de la vejez}

Hasta la aparición de la geriatría, la vejez fue considerada como un sinónimo de enfermedad y deterioro. Estas ideas eran herederas de los planteamientos decimonónicos del médico higienista Monlau. Según estos, desde el punto de vista físico y moral, la vejez se identificaba con atrofia y decadencia, pues Monlau defendía que «la vejez es, ya de por sí, una enfermedad» ${ }^{35}$. Como hemos indicado anteriormente, los geriatras proporcionaron una nueva interpretación de esta etapa vital y, así, se defendió también en España. El secretario de la Sociedad Española de Geriatría, Francisco Vega Díaz -en la presentación de la Semana Geriátrica - afirmó que «Envejecer no es enfermar. Envejecer es cambiar» ${ }^{36}$. Además, se empezó a considerar, tal como se había aprobado en el Congreso Internacional de Lieja, la dimensión social del envejecimiento. Prueba de ello fueron declaraciones como las de Gonzalo Piédrola Gil, llamado en 1955 a dirigir la Sección de Gerocultura y Geriatría creada por la Dirección General de Sanidad:

«Si la medicina es en la actualidad Ciencia Médica y Social, a la vez que arte, en el momento presente ha de prestar gran atención a la vejez, que no es enfermedad, sino fenómeno natural, inevitable y biológico, en el que se presentan numerosos problemas de tipo médico, sanitario, sociológico, económico y cultural» 37 .

Como ya hemos indicado inicialmente, esta evolución en torno a la investigación de la vejez es fundamental para encuadrar los paulatinos cambios sociales y políticos de las siguientes décadas. En la prensa podemos rastrear el impacto de esta creciente actividad científica en torno al proceso de envejecimiento y la asimilación de algunos de los nuevos conceptos ${ }^{38}$. Por

\footnotetext{
35. Arquiola, n. 26, p. 8.

36. También es significativa la puntualización de Beltrán Báguena en el prólogo de su obra Prevención de la vejez, achacosa y cuidados de los ancianos: «envejecer no es una desgracia, sino una gran fortuna que no está reservada a todos los que nacen». Sin embargo, el mismo Beltrán Báguena demostró sus dudas, expuestas en la Semana Geriátrica, donde afirmó que «el nudo gordiano» seguía siendo «determinar si la vejez es una enfermedad». Respectivamente Arquiola, n. 26, p. 13 y Arquiola Llopis, Elvira. La vejez a debate. Madrid: Consejo Superior de Investigaciones Científicas; 1995, p. 170.

37. Piédrola Gil, Gonzalo. El envejecimiento de las poblaciones. Madrid: Dirección General de Sanidad; 1955, p. 36.

38. En una entrevista al doctor Carlos Blanco Soler se pudo leer: «Entonces, incluso cuando la vejez ha llegado, ¿hay remedios para curarla? Para eso está precisamente la "geriatría" rama de la
} 
ejemplo, en mayo de 1963, el semanal Blanco y Negro inició la publicación de una serie de artículos en los que, bajo el título «Como vivir mucho y siempre joven», se alegaba lo siguiente:

«Para nosotros la vejez es sinónimo de enfermedad, de debilidad. Esta manera de pensar está formada por espectros heredados de otra época en que el ser humano se sentía entregado al azar y a una multitud de peligros (...) Pero, poco a poco, la naturaleza, que en otros tiempos dominaba al hombre, se ha convertido en objeto para el hombre (...) cuando el envejecimiento se transforme a su vez en objeto, dejará de dar miedo» ${ }^{39}$.

El objetivo principal de esta serie fue la recopilación de indicaciones para retrasar el envejecimiento. Entre ellas destacan los consejos en torno al cuidado del cuerpo, pero también se empezó a hacer hincapié en la importancia de la felicidad y en la necesidad de seguir socialmente activo durante la vejez. La ampliación de la esperanza de vida empezaba a ser ya un hecho gracias al desarrollo de la "todopoderosa" ciencia y las ventajas de la modernidad. Por ende, los antiguos modos y costumbres sociales vinculadas con la vida durante la vejez empiezan cambiar ${ }^{40}$. Muy significativas en este sentido, aunque no representativas, son las declaraciones del médico Carlos Blanco Soler:

«Hoy día la vejez, prácticamente, ha desaparecido. Todos los hoteles de turismo, las estaciones de placer, los trenes de lujo, están llenos de matrimonios jubilados que disfrutan de un bien ganado descanso luego de muchos años de trabajo y actividad fructífera. Hace un siglo se imponía la necesidad de ser viejo, las mujeres consideraban que su vida activa había terminado antes de los cuarenta años, e incluso muchas veces el ciclo de su vida de mujer terminaba efectivamente hacia esa edad. Hoy, en cambio, se impone la necesidad

medicina que se ocupa de los viejos, de las enfermedades debidas a la vejez, del agotamiento». García-Diego, Begoña. Queremos envejecer lo más tarde posible. Blanco y Negro. 9 Dic 1961: 106.

39. Como vivir mucho y siempre joven. Blanco y Negro. 8 Jun 1963: 12.

40. Según un artículo: «Debemos, pues envejecer, y que no nos pese, pero las mujeres de hoy tenemos la enorme suerte de hacerlo mucho más tarde que nuestras abuelas». García-Diego, n. 38, p. 106. Sobre la longevidad, véase, por ejemplo, el siguiente fragmento: «Pero parece que ahora va en serio; parece que, de verdad, nos aproximamos, a la edad dorada en que Adán y Eva estrenarán, a los 50, 60 y 70 años, una segunda primavera. (...) No se trata, por supuesto, de ningún afrodisíaco, sino de un auténtico «tratamiento» químico-biológico con el cual la ciencia parece haber conseguido (...) «rejuvenecer» y «revitalizar» considerablemente el cuerpo y la mente». ¿Qué edad tiene hoy la mujer de treinta años? Blanco y Negro. 11 Nov 1967: 117. 
de no envejecer. Las damas de cincuenta años siguen en la brecha, despiertan de su juventud considerablemente aumentada por la experiencia de los años. Hombres mayores se casan con muchachas jóvenes y las hacen muy felices. Es el signo, magnífico, de nuestra época» ${ }^{41}$.

Tal como vemos, los postulados de la geriatría despertaron la curiosidad de los medios en tanto que la mostraban como una disciplina innovadora que prometía alargar la vida. Pero la geriatría iría más allá y su desarrollo y puesta en práctica, irremediablemente la llevarían a un consecuente análisis de la situación socioeconómica de las personas mayores. De hecho, el estudio de la vejez impulsado por los geriatras les encaminó rápidamente hacia una reflexión en torno a las graves carencias sociales del momento. En 1955, Piédrola Gil señaló que:

«en la ayuda a la vejez hay que considerar no sólo los aspectos médicos, sino los sociales y hasta los psicológicos, y es que todo anciano, por el hecho de serlo, debe poseer un hogar donde guarecerse, una pensión lo suficiente para vivir y sentirse independiente, asistencia médica gratuita y, en fin, no debe sentirse ni aislado ni solo» ${ }^{42}$.

Por otra parte, se empezó a difundir un discurso que señalaba que la atención a las personas mayores no solo era una obligación cristiana, sino sobre todo un deber social, una acción de recompensa y por tanto de justicia por el trabajo desempeñado. El propio Alberto Salgado Alba manifestó —en un documento publicado en 1966 por el Ministerio de Gobernación- lo siguiente:

«Si bien es cierto, la sociedad tiene y debe pensar que, si aumenta la población pasiva, con los inconvenientes económicos que hemos apuntado anteriormente, también es cierto que, gracias al esfuerzo, al trabajo y al ahorro de esta población pasiva, de estos ancianos, se ha podido llegar al estado actual de adelanto y bienestar que hoy disfruta la sociedad, con relación a las generaciones pasadas. La sociedad tiene, por tanto, para con los ancianos unas

41. García-Diego, n. 38, p. 106. Sin embargo, no todas las personas vieron con el mismo entusiasmo el alargamiento de la vida. Así, en un artículo de opinión se alertaba: «Sin embargo, este gran estirón en el número de años de la vida plantea no pocos problemas ascéticos y morales que yo, la verdad, no veo muy estudiados». Federico Sopeña, P. Meditación sobre los viejos. Blanco y Negro. 14 Ene 1961: 109.

42. Piédrola, n. 37, p. 57. 
obligaciones no sólo de tipo puramente moral, sino por reciprocidad obligada de tipo puramente económico» ${ }^{43}$.

La misma idea la hemos encontrado en revistas específicas como Documentación Social e incluso en la propia prensa generalista, ya sea en columnas de opinión o cartas del lector ${ }^{44}$. Esta evolución en la opinión publicada coincide en lo político con el desarrollo del segundo franquismo y su intento de poner en marcha una seguridad social que por un lado afrontase las profundas carencias del sistema, y por el otro, también avalara al régimen en su proceso de legitimización ante Europa. Sin embargo, ninguna de las medidas desarrolladas por el franquismo fue capaz de aplacar el creciente espíritu crítico y el repunte de las movilizaciones sociales. Ya en 1971, jubilados y pensionistas asturianos protagonizarían los primeros encierros reivindicando mejoras económicas y asistenciales ${ }^{45}$. Protestas que, en los años de la transición, tendrán una importante influencia sobre la extensión de las pensiones de la Seguridad Social.

\section{La institucionalización de la geriatría y su desarrollo político en el segundo franquismo}

La evolución política, económica y social del segundo periodo franquista favoreció que las propuestas geriátricas pasasen del ámbito puramente académico y profesional al institucional. Para el aspecto que nos ocupa jugó un papel importante el ya citado aumento de la esperanza de vida, que empezó a ser significativa a partir de los años cincuenta ${ }^{46}$. Otra cuestión importante fue la mejora de la atención sanitaria general pues permitió a los agentes implicados concentrarse en nuevos frentes de actuación ${ }^{47}$. Así, Cáritas señaló

\footnotetext{
43. Salgado, n. 16, p. 180.

44. Véase, por ejemplo, el siguiente artículo: Alegre, Santiago. Recursos de la vejez. Documentación Social, 1963; 20: 36.

45. Martos Contreras, n. 19, p. 631-638. En 1971 un 85\% de los pensionistas consideraban que su pensión era bastante o totalmente insuficiente. VVAA. Informe Gaur. La situación del anciano en España. Madrid: Fondo para la investigación económica y social de la Confederación Española de Cajas de Ahorras; p. 306.

46. La esperanza de vida, tras cumplir los 60 años, aumentó a lo largo del siglo XXI de la siguiente manera: en 19002.49 años, en 195016.55 años y en 197018.57 años. VVAA, n. 20, p. 468.

47. Rodríguez Ocaña, Esteban; Martínez Navarro, Ferrán. Salud Pública en España. De la Edad Media al siglo XXI. Sevilla: EASP, 2008, p. 91-98.
} 
«la ancianidad como problema de nuestro tiempo» y en 1959 creó la Sección de Asistencia a los Ancianos. En 1962, en su Asamblea Nacional, describió la «ancianidad» como uno de los problemas sociales más acuciantes en España $^{48}$. La Cruz Roja también eligió a las personas mayores como uno de sus principales campos de actuación, poniéndose al frente, como ya vimos, de las instituciones más novedosas ${ }^{49}$. Por otra parte, el Auxilio Social había empezado a transformar, desde principios de la recuperación económica, sus ya citados comedores en «clubs de ancianos» ${ }^{50}$.

En cuanto a las políticas públicas, en 1955 el director general de Sanidad, José Alberto Palanca Martínez-Fortún, fundó dentro de la Dirección General de Sanidad la Sección de Gerocultura y Geriatría, a cuyo frente colocó al renombrado médico militar Gonzalo Piédrola Gil ${ }^{51}$. Varios años después, en 1961, el médico Blanco Soler, entonces director del Servicio Geriátrico del Hospital de la Cruz Roja - en un discurso dirigido a la Real Academia de Medicina - aportó un balance sobre los avances de la Geriatría y solicitó la creación de dispensarios geriátricos. Su intervención recibió la respuesta del mismo Alberto Palanca - presidente de la Academia y, como ya indicamos, director general de Sanidad-comprometiéndose a promover la creación de dispensarios en las Jefaturas Provinciales de Sanidad ${ }^{52}$. De hecho, poco después, el jefe de la Sección de Gerocultura y Geriatría, Píedrola Gil, encabezó el proyecto, Lucha contra la vejez, donde se preveía la creación de

48. Sánchez Jiménez, José. Cáritas española: 1942-1997: acción social y compromiso cristiano. Madrid: Cáritas Española; 1998, p. 161 y 106.

49. Martos Contreras, Emilia. Proyectos sociales en la transición a la democracia: El Hospital Geriátrico de Almería. In: Fernández Amador, Mónica; Martos Contreras, Emilia, eds. Democracia y Mundo rural. Almería: Universidad de Almería; 2017.

50. Vista la cada vez mayor presencia de personas mayores, ideó una especie de centros sociales en los que se ofrecía comida a un precio asequible. Estos clubs se convirtieron en poco tiempo en centros de reunión, con el bar como único y principal atractivo. Fueron frecuentados fundamentalmente por hombres, ya que la ideología de la época no concebía la presencia de mujeres en este tipo de espacios.

51. Gonzalo Piédrola Gil tuvo influencia sobre otras muchas áreas de la medicina desde su puesto como catedrático en diversas disciplinas y universidades españolas. Entre sus numerosas publicaciones destaca su trabajo sobre Medicina Preventiva y Salud Pública. Su discurso de ingreso como Académico de honor trato precisamente sobre el envejecimiento. Piédrola Gil, Gonzalo. El arte de envejecer: discurso como Académico de honor del Exmo. Sr. Prof. G. Piédrola Gil. Actualidad Médica. 1991; 720: 571-604.

52. Piédrola Gil, Gonzalo. Glosa y comentarios al año Internacional de la Tercera Edad. Anales de la Real Academia Nacional de Medicina. 1982; 99: 663. 
dichos dispensarios ${ }^{53}$. Sin embargo, como frecuentemente sucedía durante el franquismo, el proyecto no llegó a tener repercusión. Entre las principales causas de este fracaso se encontró la ausencia de un verdadero respaldo económico y profesional. En 1976, Francisco Jiménez Herrero, quién había sido director general de Sanidad, declaró que «la lucha contra la vejez» no

«se había extendido en España más que a media docena de Jefaturas Provinciales de Sanidad con más intención de ensayo piloto que de ejecución seria ambiciosa, por la falta de medios y personalidades médicas que la apoyasen con empuje decidido» ${ }^{54}$.

Esta falta de resolución estuvo agravada por el enfrentamiento entre la Dirección General de Sanidad y las fuerzas en torno al Seguro de Enfermedad. Así, mientras desde el Ministerio de Gobernación se propuso la «Lucha contra la Vejez», desde el Ministerio de Trabajo se impulsó la Seguridad Social y se apoyó el «Plan Gerontológico». Por lo tanto, como ocurrió en otros aspectos de la sanidad, el ámbito de la vejez sufrió la duplicidad y la descoordinación resultante del enfrentamiento entre las llamadas familias del franquismo. Una pugna que se saldó con la «victoria» de las propuestas surgidas desde el Ministerio de Trabajo y la previsión social ${ }^{55}$.

La ley de Bases de 1963 extendió la Seguridad Social hacia nuevos campos de promoción social y comunitaria. Una de sus principales mejoras fue el impulso a la asistencia social, lo que se tradujo esencialmente en la proliferación de centros sanitarios, aunque también contempló la creación de otro tipo de instituciones ${ }^{56}$. Así, desde finales de la década de los sesenta, se empezaron a crear residencias públicas. Estas residencias suponían una gran renovación en el ámbito que nos ocupa, tanto por sus nuevas construcciones como por su sistema de funcionamiento. Pese a todo, aparte de ser clara-

53. También hubo propuestas a nivel municipal, como el Plan Gerontológico Municipal de Madrid, que contemplaba una serie de acciones complementarias como la creación de unidades geriátricas de larga estancia. Piédrola, n. 37, p. 665.

54. Jiménez Herrero, Fernando. 1926-1976: cincuenta años de la geriatría en España. Gaceta Médica Española. 1976; 8: 379.

55. Molero; Jiménez, n. 4, p. 71.

56. En el propio I Plan de Desarrollo Económico y Social, para 1964-1967, se propuso como objetivo intensificar la «acción de ayuda a la ancianidad». Plan de Desarrollo Económico y social para el período, 1964-1967. BOE. 3 Feb 1964. Véase Durán Villa, Francisco. Los servicios sociales especializados para la vejez. In: WVAA. SEMATA 18. Vejez y envejecimiento en Europa Occidental. Santiago de Compostela: Universidad de Santiago de Compostela; 2006, p. 213-214. 
mente insuficientes, solo acogían a los llamados «ancianos válidos», es decir, personas mayores no dependientes. Esto resultaba desfasado en un periodo en el que la evolución de la geriatría ya estaba planteando la importancia de favorecer la permanencia de las personas mayores en su contexto social e insistía en la creación de servicios como el hospital de día o la ayuda a domicilio $^{57}$. Además, los centros más necesarios eran aquellos que pudiesen atender de forma específica a las personas mayores con necesidades de cuidados intensivos. Nos referimos a las que en la época eran calificadas como plazas para «no válidos» y que precisamente no estaban contempladas en las nuevas residencias de la Seguridad Social.

A finales de los sesenta las Mutualidades Laborales se pusieron a la cabeza al crear una comisión permanente que elaboró el llamado Plan Gerontológico de las Mutualidades Laborales. El documento, publicado en 1969, consideró la situación social de las personas mayores y criticó las graves carencias del sistema asistencial; convirtiéndose así en el impulso político más importante en el campo de la protección de las personas mayores. Aunque defendió que «al anciano ha de mantenérsele en su hogar, rodeado del ambiente que le es familiar, prestándole el apoyo necesario mediante la ayuda a domicilio y los «clubs», desarrolló ampliamente la propuesta de creación de instituciones asistenciales de acogida ${ }^{58}$. En general, y tal como señala Campos Egozcue, el Plan Gerontológico de las Mutualidades Laborales - a pesar de contemplar la vejez desde una óptica paternalista-, expresó la toma de conciencia ante las transformaciones socioeconómicas del país y señaló la necesidad urgente de modernizar los sistemas de atención a la población mayor ${ }^{59}$. El plan indicó expresamente que, dentro del ámbito de la vejez, «la asistencia social aparece como el gran vacío a cubrir» y criticó claramente «la gestión de la protección de la vejez, la desatención pública del sector y la ineficacia en este campo de los Planes de Desarrollo» ${ }^{60}$.

57. En 1970 el Consejo Europeo recogió esta recomendación en una resolución específica. Consejo de Europa, Comité de Ministros. Sobre la protección social y médico-social a la vejez. Bruselas, 1970, p. 6-7, citado en Alonso Torréns, Francisco Javier dir. La ancianidad en Huelva. Madrid: Cares; 1977, p. 195.

58. Mutualidades Laborales. Plan Gerontológico de ML. Madrid: 1969, p. 31, citado en Campos Egozcue, Begoña. La construcción de una política social de vejez en España: del franquismo a la normalización democrática. Revista Española de Estudios Sociológicos. 1996; 73: 247.

59. Campos, n. 58, p. 247.

60. Sánchez, n. 48, p. 106. 
La propuesta de las Mutualidades Laborales tuvo una buena acogida por parte del Ministerio de Trabajo ${ }^{61}$. El nuevo ministro, Licinio de la Fuente, encargó a Enrique de la Mata - anterior presidente de la Asamblea de la Cruz Roja y ahora director General de la Seguridad Social- la elaboración de un Plan Gerontológico Nacional. La dirección del proyecto recayó sobre Juan Vidal Hernández, un alto cargo del Mutualismo Laboral, y la coordinación en el doctor Alberto Salgado Alba, quién en estos momentos ya ejercía de secretario de la Sociedad Española de Geriatría y Gerontología y jefe del Servicio de Geriatría del Hospital Central de la Cruz Roja de Madrid ${ }^{62}$. Como vemos, el proyecto no sólo nació bajo el abrigo de las instituciones españolas más punteras en ese ámbito, sino que contó con la supervisión del mayor experto en el área, aunque sin contar con la Sección de Gerocultura y Geriatría de la Dirección General de Sanidad. El 19 de marzo de 1970, se puso en marcha el Plan Gerontológico, creándose el Servicio Social de Asistencia a los Ancianos, antecedente directo del Instituto Social de Servicios Sociales (Inserso $)^{63}$. Este Servicio Social de Asistencia a los Ancianos, dependiente de la Seguridad Social, se marcó como objetivo principal «compensar el déficit asistencial en la protección social pública de los ancianos españoles integrados en el sistema contributivo». Según la norma, era conveniente dar un carácter general y planificado a las acciones parciales de asistencia que ya estaban realizando algunas mutualidades. Dentro de las acciones que contemplaba, se encontraba la creación y mantenimiento de centros gerontológicos y la organización de asistencia o ayuda domiciliaria, además de otras actividades complementarias ${ }^{64}$. Su principal originalidad radicó,

61. De hecho, ya en unas jornadas mutualistas en Barcelona, en 1969, el entonces ministro de trabajo, Romeo Gorría, prometió: «Vamos a iniciar la puesta en marcha de un Plan Gerontológico Nacional (...) y una de sus primeras realizaciones será la residencia de Ancianos de Barcelona. También será dotada la ciudad de un primer Hogar del Jubilado, instalado en la futura Delegación de las Mutualidades Laborales». Congreso mutualista. La Vanguardia, 8 Jul 1969: 33.

62. Martínez Gómez, José Manuel. Los servicios sociales en España. La influencia de Alberto Salgado en su desarrollo. Revista Española de Geriatría y Gerontología. 2001; 36: 65.

63. Martos Contreras, Emilia. Transición y política social: El Instituto Nacional de Servicios Sociales (Inserso). In: Quirosa-Cheyrouze y Muñoz, Rafael; Fernández Amador, Mónica y eds. Poder y Transición en España. Las Instituciones políticas en el proceso democratizador. Madrid: Biblioteca Nueva; 2017, p. 181-192.

64. BOE. 23 Mar 1970 y Plan Nacional de Seguridad Social de Asistencia a Ancianos, BOE. 3 Mar 1971. Sobre la puesta en marcha del Plan General de Asistencia a Ancianos véase el reportaje del NO-DO de 1973, en el que se planteaba un panorama mucho más positivo del que fue en realidad. Según dicha emisión: «Quizá el termómetro que mida con mayor fidelidad el grado de evolución y progreso de un pueblo sea la atención que se presta en él al bienestar y cuidado de 
precisamente, en el reconocimiento gubernamental de unas necesidades más allá de las prestaciones económicas, admitiendo las críticas del Plan General de las Mutualidades Laborales. Además, según Demetrio Casado, otra novedad fue que no se trataba «tanto de socorrer al anciano mísero y/o valetudinario, como de expresar aprecio social y político al productor mediante servicios orientados a mejorar su calidad de vida» ${ }^{65}$. Con todo, el proyecto nació con limitaciones internas importantes. La primera de ellas fue que estaba enfocado solamente para las personas englobadas dentro de la Seguridad Social, que por aquel entonces aún eran una minoría ${ }^{66}$. Por lo tanto, las personas más desfavorecidas continuaban sin tener un plan de atención social, salvo la ya citada beneficencia. No obstante, el principal fallo del proyecto fue que el desarrollo real de los servicios fue muy reducido. De hecho, muchos de sus planteamientos como por ejemplo la ayuda a domicilio, nunca llegaron a materializarse ${ }^{67}$.

La Dirección General de Beneficencia y las Diputaciones Provinciales continuaron rigiendo los escasos y anticuados asilos, mientras que el Auxilio Social, a pesar de su clara decadencia, mantuvo algunos clubs y un reducido número de comedores que atendían preferentemente a personas mayores ${ }^{68}$. En realidad, las congregaciones religiosas, como en décadas anteriores, continuaron siendo las principales garantes de la asistencia a las personas mayores, sobre todo en los casos de extrema necesidad. La creación del Fondo Nacional de Asistencia Social abrió una pequeña vía para la financiación puntual de algunas instituciones, que se acogieron a sus prestaciones arbitrarias para

sus ancianos. La obligación que tenemos de cuidar a nuestros mayores se convierte en propia satisfacción al contemplar la obra que en este sentido ha iniciado el Ministerio de Trabajo por Medio del Plan General Nacional de la Seguridad Social (...) Tenemos por toda nuestra geografía hogares y residencias cuya única misión es reintegrar a la ilusión y a la convivencia a los que por su edad pudieran sentirse desplazados». NO-DO. 14 May 1973. Archivo de RTVE. Disponible en: http://www.rtve.es/filmoteca/no-do/not-1584/1469890/

65. Casado Pérez, Demetrio. Acción social y Servicios Sociales. In: VVAA. V Informe sobre la situación social en España. Madrid: Fundación FOESSA; 1994, p. 1785.

66. En 1975 había en España 5.310 .800 personas mayores de 60 años, pero tan solo 1.910.000 recibían algún tipo de pensión. Datos obtenidos de García de Blas, Antonio. La jubilación como respuesta al problema de desempleo. Revista de Seguridad Social. 1982; 16: 49 y VVAA, n. 20, p. 468.

67. Véase Medina Tornero, Manuel Enrique. Evaluación de la calidad asistencial del servicio de ayuda a domicilio. Universidad de Murcia, 2000; p. 73.

68. En noviembre de 1975 el Auxilio Social aún gestionaba 56 clubs de ancianos, cuatro residencias con capacidad para 200 personas, una ciudad de matrimonios con capacidad para 92 personas y 43 comedores para adultos. Sánchez Blanco, Laura. Auxilio Social y la educación de los pobres: del franquismo a la democracia. Foro de Educación. 2008; 1: 158. 
intentar reformar, y en los mejores casos ampliar, las deficientes infraestructura de los asilos. Sin embargo, en aquellos casos que se aprobó una colaboración económica, esta resultó ser claramente insuficiente. Por esta razón, en última instancia las donaciones continuaron siendo la principal fuente de financiación de la asistencia social religiosa ${ }^{69}$. El Fondo Nacional también contemplaba las prestaciones económicas a personas mayores necesitadas, aunque se trató de una ayuda arbitraría y muy exigua ${ }^{70}$. Según denunció el llamado Informe Gaur, a principios de los años setenta alrededor de medio millón de personas mayores sobrevivían sin ningún tipo de prestación. De ellos, el $80 \%$ eran mujeres ${ }^{71}$.

No obstante, aunque las medidas políticas fueron un fracaso, no podemos negar la evolución social del concepto de ła vejez y de los derechos de las personas mayores. Como tratamos, estos cambios ya habían empezado a sentirse a mediados de siglo, sin embargo, durante las siguientes décadas fueron en aumento. Así lo consideran también autores como Campos Egozcue, para quién las reformas de la Seguridad Social, a pesar de su ineficacia, supusieron «un punto de inflexión positivo en el ámbito de las políticas de la vejez al activar un amplio debate social que contribuyó a la redefinición de la problemática» ${ }^{72}$. De hecho, esta evolución ya había sido percibida en su propio contexto, como muestra este fragmento de 1975:

«Desde hace unos diez años ha surgido en España, bajo la influencia de corrientes de opinión generalizadas en Europa Occidental y América, un cambio de actitud hacia los ancianos, cambio que ha dado lugar a lo que nosotros denominaremos una "nueva ideología" sobre la vejez en el marco de una "nueva ideología" respecto a los llamados "marginados". (...) La vejez se ha convertido en categoría social con entidad propia. (...) El anciano se ha transformado, en la retórica de nuestra sociedad, en sujeto ${ }^{73}$.

69. Así, por ejemplo, en el caso de la provincia de Almería, que es el que hemos estudiado con mayor profundidad, el Asilo-Hogar de las Hermanitas de los Desamparados continuaba a mediados de los setenta colocando recipientes en diferentes puntos del mercado central, para recibir donaciones de alimentos. Martos Contreras, n. 19, p. 790.

70. Fue una prestación insuficiente incluso después de la subida de 1972, que la reglamentó en 1000 pesetas. BOE. 24 Jul 1972.

71. En una encuesta publicada por el mismo informe, un $13 \%$ de las mujeres, frente a un $3 \%$ de hombres, admitió no comer todo lo que necesitaban. VVAA, n. 45, p. 277 y 329.

72. Campos, n. 58, p. 247.

73. VVAA, n. 45, p. 518. 
Asimismo, el documento intuyó la importancia de la figura del «jubilado» en este cambio de la opinión pública, ya que ser «jubilado» era tener reconocida «la condición de ex-trabajador», entendido como

«los hombres que "han hecho posible nuestro presente y nuestro futuro", son "nuestros mayores", los que nos "precedieron en el camino" según el "nuevo lenguaje” (...) Sobre cualquier otro pensamiento, hoy priva el de ver la jubilación como la obligación social de conceder un descanso justamente ganado y la posibilidad de un amplio tiempo libre, en el que pueden gozar de los placeres que antes la vida les negó, a quienes han venido trabajando incansablemente hasta una edad tope» ${ }^{74}$.

En cualquier caso, el mismo informe advirtió de la contrapartida de esta opinión pública que había creado «una retórica abstracta que mitifica al viejo y a la vejez, elevándola e idealizándola tanto que pierde todo contacto, todo parecido con la vejez real y vivida por los ancianos de nuestros pueblos y ciudades. Es la deshumanización por el mito» ${ }^{75}$. El documento adjuntaba y criticaba un ejemplo extraído de la prensa:

«Ante esa puerta nos encontramos, miradla como un arco iris en un horizonte abierto. Atrás quedó la tormenta de una vida intensa y activa con truenos y relámpagos de felicidad y desgracia; ahora renace la calma, con olor a tierra mojada y luz policromada de atardecer... Hemos de confesar que nada de esto hemos encontrado entre los ancianos con los que hemos hablado, ni posesión de la verdad, ni desarrollo de aficiones latentes, ni olores a tierra mojada, ni luces policromadas, sino pobreza, analfabetismo, fatiga, incapacidad» ${ }^{76}$.

\section{Conclusiones}

Durante los años del franquismo la situación de un importante número de personas mayores fue bastante complicada. Los índices de pobreza y de desatención eran tan elevados que, desde mediados de los años cincuenta, se convirtieron en la principal preocupación de las instituciones asistenciales. Sin duda, esta preocupación no se forjó en España, pues se planteó en diversos eventos internacionales. Sin embargo, en el caso español, el estan-

\footnotetext{
74. El Diario Vasco. 20 Nov 1970, citado en VVAA, n. 45, p. 518.

75. VVAA, n. 45, p. 518.

76. VVAA, n. 45, p. 518.
} 
camiento socio-político supuso un importante agravante. Esto no significa que la dictadura no aprobase medidas relacionadas con la protección de las personas mayores. De hecho, desde el principio, e influido por los latentes planteamientos fascistas, avanzó en el desarrollo de los seguros sociales. En los siguientes años, las instituciones franquistas asumieron otras mejoras, como la creación de la Sección de Gerocultura y Geriatría o la implantación de los servicios sociales, con planes como la Lucha contra la Vejez o el Plan General Gerontológico Nacional.

Sin embargo, como se ha visto, ninguna de estas propuestas vino a solucionar los problemas de las personas mayores en España. Este fracaso se debió tanto a la falta de compromiso como a la duplicidad y descoordinación causada por los enfrentamientos entre los diferentes ministerios. Para los años setenta, la pobreza del colectivo continuaba siendo una realidad indiscutible y así lo demostraron con datos los primeros informes sociológicos de la época. De hecho, estas carencias no solo se daban en un amplio número de personas sin protección social, sino que también fueron una constante entre los propios pensionistas, cuya prestación era en la mayoría de los casos alarmantemente exigua.

En cuanto a la atención asistencial, y a pesar de la paulatina creación de residencias, el Estado franquista no aprovechó la pronta introducción de la geriatría para buscar soluciones. A finales del franquismo las instituciones caritativas tradicionales, en su mayoría con infraestructuras y planteamientos completamente desfasados, seguían siendo el lugar donde acudían las personas mayores necesitadas. Entre ellas, las mujeres fueron las más afectadas, tanto porque eran mayoría como por el hecho de que tenían menos acceso a las pensiones. Su tradicional dedicación a las labores no remuneradas, respaldada por las políticas de género franquistas, las dejaba completamente desprotegidas cuando los recursos económicos o familiares fallaban.

Por lo tanto, a lo largo de las cuatro décadas de la dictadura, la situación real de las personas mayores no cambió mucho. Sin embargo, si hemos podido rastrear una significativa evolución en el concepto de la vejez. Este fenómeno, que se dio a nivel internacional, se nutrió de múltiples causas. Principalmente, el aumento de la esperanza de vida. Pero fue la evolución de la medicina que se estaba gestando desde comienzos del siglo XX lo que llevaría a la creación de una materia específica dedicada a las personas mayores. Sin duda, la propia aparición de la geriatría fue una clara señal de los cambios que se estaban gestando. La nueva disciplina supuso un paso adelante, ya que su evolución conceptual y metodológica demostró que un 
tratamiento adecuado podía suponer una mejora cualitativa importante para las personas mayores. Así, el optimismo de la época hizo soñar a muchos con la eterna juventud, mientras que el pujante sistema capitalista vio en el afán por la juventud eterna un gran negocio ${ }^{77}$. Además, la extensión de los seguros sociales estaba creando un nuevo nicho de mercado, el de los jubilados, que abrazaron los nuevos consejos de «revitalizarse» y se convirtieron en importantes consumidores de nuevos sectores como el turístico.

A España también llegaron estos cambios, aunque su realidad política y económica determinó su evolución. Es llamativa la temprana asimilación de la geriatría y el importante esfuerzo de algunos profesionales por su implantación. Sin embargo, al igual que ocurrió en el extranjero, el poner la mirada sobre las personas mayores hizo remarcar una realidad deprimente. Esto favoreció a que se forjara un discurso crítico que, además, instalado dentro de los sectores minoritarios de la atención médica y social, pudo burlar la censura de la época. No nos cuesta trabajo encontrar documentos de la época en los que se señala claramente las múltiples deficiencias. De hecho, el propio sistema dictatorial asumió estas graves carencias, y así lo dejó patente con la asunción de proyectos de mejora. Sin embargo, como ya dijimos, una cosa era asumir la necesidad de mejoras, y otra diferente hacer los cambios necesarios para una evolución efectiva. En consecuencia, se creó una creciente asimetría entre la realidad y las promesas del franquismo. Queda para investigaciones posteriores profundizar en la influencia de este desequilibrio y su peso en la creciente actitud crítica de los colectivos de jubilados y pensionistas.

77. «Pero parece que ahora va en serio; parece que, de verdad, nos aproximamos a la edad dorada en que Adán y Eva estrenaran, a los 50, 60 y 70 años, una segunda primavera». Blanco y Negro. 11 Nov 1967: 16. 
